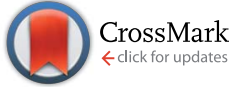

Cite this: RSC Adv., 2015, 5, 49363

Received 5th May 2015

Accepted 22nd May 2015

DOI: $10.1039 / \mathrm{c} 5 \mathrm{ra0} 8302 \mathrm{~h}$

www.rsc.org/advances

\title{
Two-photon absorption of polyfluorene aggregates stabilized by insulin amyloid fibrils $\uparrow$
}

\author{
P. Hanczyc, ${ }^{\text {,ab }}$ A. Justyniarski, ${ }^{c}$ D. A. Gedefaw, ${ }^{\text {b }}$ M. R. Andersson, ${ }^{\text {bd }}$ M. Samoc ${ }^{c}$ \\ and C. Müller ${ }^{b}$
}

\begin{abstract}
We report on the photophysical and optical properties of a polyfluorene derivative (PFO) and its binding to the amyloid-forming protein insulin. The complexation is based on weak supramolecular interactions between amyloid fibrils and PFO in dissolved and aggregated forms. In particular, complexes of polyfluorene aggregates display sizeable two-photon absorption when stabilized by insulin fibril nanotemplates. We propose that a two-photon absorption enhancement mechanism is related to the combination of through-bond and through-space interactions of PFO. The findings reported here suggest that polyfluorene aggregates offer good nonlinear absorption properties and can be used as molecular markers for amyloid fibril recognition.
\end{abstract}

\section{Introduction}

Conjugated polymers have attracted much attention as effective probes for amyloid protein fibril recognition and are being developed for imaging, diagnostic and therapeutic applications ${ }^{1}$. Their tunable photophysical properties in the visible and near-infrared spectrum, which is particularly sensitive to protein conformation upon binding, allow for monitoring of the fibrillization with a variety of optical techniques ${ }^{2}$. Among those, techniques based on nonlinear optics are especially promising as they can improve penetration depth, reduce photo-bleaching and minimize photo-damage of biological systems. However, in order to use multiphoton based techniques molecules with large two-photon absorption cross-sections are required that enable efficient excitation in the near infrared region, which is safe for biomolecules. Polyfluorenes (PFO) have the potential to be interesting markers since they exhibit sizeable multiphoton absorption properties, ${ }^{3}$ which may facilitate amyloid fibril detection and imaging by less invasive multiphoton optical methods. ${ }^{4}$ In the context of binding to biomolecules, two groups of compounds are of particular interest: water-soluble conjugated polyelectrolytes and non-water-soluble conjugated polymers or oligomers. In case of the first group the functional

${ }^{a}$ University of California, Center for Oligomers \& Organic Solids, 2520A Physical Sciences Building North, Santa Barbara, USA.E-mail: hanczyc@physics.ucsb.edu

${ }^{b}$ Chalmers University of Technology, Department of Chemistry and Chemical Engineering, Kemivägen 10, Gothenburg, 41296, Sweden

${ }^{c}$ Wroclaw University of Technology, Advanced Materials Engineering and Modelling Group, Faculty of Chemistry, 50-370, Wroclaw, Poland

${ }^{d}$ University of South Australia, Ian Wark Research Institute, Mawson Lakes, South Australia 5095, Australia

† Electronic supplementary information (ESI) available. See DOI: 10.1039/c5ra08302h charged groups enhance the interaction with amyloids due to electrostatic binding with charged residues in the protofilaments of the fibrils ${ }^{5}$, whereas for the second group only weaker (stacking type) interaction occurs that is sensitive to small structural changes and the mode of interactions with the fibrils.

In this letter we explore complexation and the mechanism of molecular interactions of a non-water-soluble polyfluorene derivative composed of a backbone with ethylene glycol side chains (Fig. 1a) by investigating its photophysical properties and interaction with amyloid fibrils, most likely mediated by weak forces such as van der Waals and $\pi$-stacking.6. Two forms of polyfluorene derivative were studied: (i) PFO dissolved in ethanol and (ii) PFO that has aggregated upon addition of waterbased buffer. Then we studied the optical signatures of both forms of the polyfluorene derivative with insulin protein that is known to self-assemble into amyloid fibrils under denaturing conditions such as low $\mathrm{pH}$ and high temperature. Insulin fibrils predominantly consist of $\beta$-sheet structures and resemble the structures of diseases related proteins such as tau, A $\beta$ (1-42) (Alzheimer's) and $\alpha$-synuclein (Parkinson's) ${ }^{7}$ or prions (Creutzfeldt-Jakob's). ${ }^{8}$ The key finding of our work is that PFO aggregation can be stabilized in solution upon binding to insulin amyloid fibrils and that can lead to a significant enhancement of two-photon absorption.

\section{Experimental section}

\section{Materials}

Polyfluorene derivative with polyethylene glycol side chains (PFO) was synthesized as follows: 2,7-dibromo-9,9-bis(2-(2-methoxy ethoxy)ethyl)-9H-fluorene (0.264 g, $0.5 \mathrm{mmol}), 2,2^{\prime}-(9,9-\mathrm{bis}(2-(2-$ methoxyethoxy)ethyl)-9H-fluorene-2,7-diyl)bis(4,4,5,5-tetramethyl1,3,2-dioxaborolane) (0.311 g, $0.5 \mathrm{mmol}), \mathrm{K}_{3} \mathrm{PO}_{4}$ tribasic (1.06 g, 
(a)

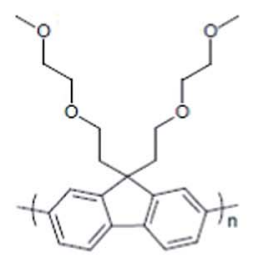

(b)

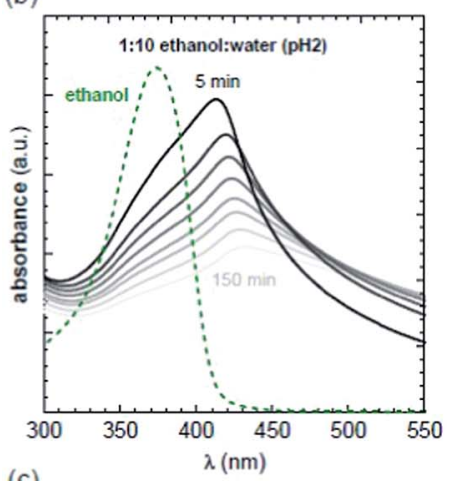

(c)

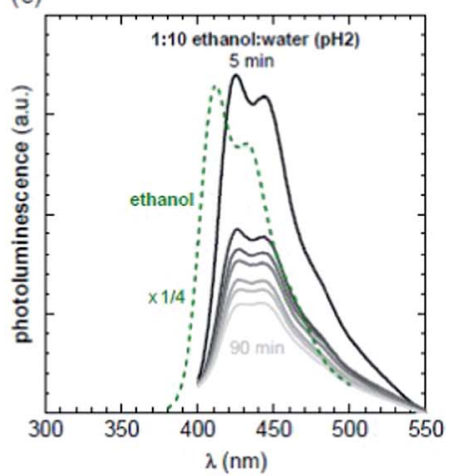

Fig. 1 Structure of the polyfluorene derivative (PFO) (a); absorption of PFO in ethanol (green dashed) and after addition of $\mathrm{pH}=2$ buffer as function of time (b); photoluminescence spectra of PFO excited at $375 \mathrm{~nm}$ after adding $\mathrm{pH}=2$ buffer as a function of time (c).

$5 \mathrm{mmol})$, aliquot 336 (3 drops) and water $(2.3 \mathrm{~mL})$ were dissolved in toluene $(15 \mathrm{~mL})$ and nitrogen gas was bubbled for 20 minutes. $\mathrm{Pd}_{2}(\mathrm{dba})_{3}(9 \mathrm{mg}, 0.01 \mathrm{mmol})$ and $\mathrm{P}(o \text {-tolyl })_{3}(12 \mathrm{mg}, 0.04 \mathrm{mmol})$ were added to the reaction mixture and nitrogen gas bubbling continued for an additional 25 minutes. The reaction mixture was heated at $90^{\circ} \mathrm{C}$ for 1 hour and 20 minutes. The oligomer solution was precipitated by drop-wise addition of hexane and the solid was collected by filtration. The oligomer was re-dissolved in THF and passed through short silica gel column using THF as eluent. The oligomer solution was concentrated on a rotavapor and precipitated by drop-wise addition of hexane and the solid was collected by filtration. The oligomer was further purified by Soxhlet extraction with hexane and then washed out with THF. The volume of the THF extract was reduced by evaporation and precipitated with hexane. The solid collected was dried at $40{ }^{\circ} \mathrm{C}$ in a vacuum oven for an overnight to give a yellowish powder $(0.3 \mathrm{~g}$, $81 \%)$. The synthesis scheme is presented in Fig. S1 $†$ together with NMR spectra (Fig. S2 and S3†). Characterization by gel permeation chromatography (GPC) indicates that PFO has low numberaverage molecular weight of $\mathrm{Mn} \sim 3.3 \mathrm{~kg} \mathrm{~mol}{ }^{-1}$ and a high polydispersity index PDI $\sim 6.2$. In average there is 9 repeating units within the oligomer chain.

Amyloid fibrils were prepared as follows: monomer insulin protein from bovine pancreas was purchased from Sigma Aldrich, dissolved in $\mathrm{pH}=2(0.01 \mathrm{M} \mathrm{HCl})$ water buffer and adjusted to final concentration of $5 \mathrm{mg} \mathrm{mL}^{-1}$ and used without further purification. Prepared solutions were then heated at $65^{\circ} \mathrm{C}$ for 24 hours. Fibrils formation was tested by Thioflavine $\mathrm{T}$ (ThT) standard with monomer proteins as references (Fig. S4 $\dagger$ ).

Amyloid fibrils-oligomer complexes were prepared as follows: lyophilized polyfluorene was partially dissolved in pure ethanol and concentration was calculated to be approximately $2 \mathrm{~g} \mathrm{~L}^{-1}$ (molar extinction coefficient in ethanol $\varepsilon=4500$ ). In a next step polyfluorene was either added directly from ethanol to solution with insulin fibrils and further studied with absorption techniques or it was mixed in volume ratio $1: 10$ with water buffers first in order to initiate formation of aggregates.

\section{Methods}

Size exclusion chromatography (SEC) was performed on Waters 510 HPLC pump with a Waters 486 UV detector $(254 \mathrm{~nm})$. The system runs at room temperature with THF as eluent, $0.5 \mathrm{~mL}$ $\min ^{-1}$. The column is a Agilent PLgel $5 \mu \mathrm{m}$ MIXED-C. The concentration of the sample was $0.5 \mathrm{mg} \mathrm{mL} \mathrm{m}^{-1}$, which was filtered (filter: $0.45 \mu \mathrm{m}$ ) prior to the analysis. The relative molecular masses were calculated by calibration relative to polystyrene standards.

${ }^{1} \mathrm{H}$ NMR and ${ }^{13} \mathrm{C}$ NMR spectra were acquired from a Varian Inova $400 \mathrm{MHz}$ NMR spectrometer. Tetramethylsilane was used as an internal reference with deuterated chloroform as solvent.

UV-Vis spectroscopy. Absorption spectra were recorded on a Jasco V-670 spectrophotometer, using solvent as a baseline. Linear dichroism spectra were recorded on a Chirascan CD spectrophotometer equipped with an LD accessory unit.

Fluorescence was studied using a Hitachi F-4500 fluorescence spectrophotometer. The samples were excited at $\lambda_{\text {ex }}=375 \mathrm{~nm}$ where the maximum of absorption of PFO was in ethanol and emission was recorded in spectral range from $390 \mathrm{~nm}$ to $650 \mathrm{~nm}$.

Z-scans for two-photon absorption (TPA). The Z-scan technique is used to measure nonlinear refractive index $\left(n_{2}\right)$ and nonlinear absorption coefficient $(\beta)$. Both quantities can be measured simultaneously by monitoring changes in transmittance of a focused laser beam as the sample travels in the $z$ direction. In order to measure TPA that is attributed only to PFO-bound to amyloid fibrils first the detection limit for dissolved PFO was experimentally determined by gradual lowering of oligomer concentration by diluting it in ethanol. No open aperture (OA) signal was detectable around $0.5 \mathrm{mg} \mathrm{mL}^{-1}$ and such concentration of PFO was used for further experiments on TPA with amyloid fibrils. Samples for measuring nonlinear absorption were mixed in volume ratio 1:4 (insulin amyloid : polyfluorene) where insulin fibrils stock solution was used (5 mg mL $\mathrm{mL}^{-1}$ ) and polyfluorene at concentration $0.5 \mathrm{mg} \mathrm{mL}{ }^{-1}$. Obtained results on the cells with PFO, insulin fibrils with PFO and insulin fibrils with aggregates of PFO were calibrated against closed aperture Z-scan measurements performed on a 
fused silica plate and compared with the measurements on an identical glass cell filled with the solvents alone: mixture of ethanol/pH $=2$ water buffer for simultaneous recording of standard open-aperture (OA). The traces of OA scans obtained by dividing each of them by the laser input reference were analyzed with the help of a custom fitting program.

\section{Results and discussion}

In a first set of experiments the photophysical properties of PFO were investigated by absorption and fluorescence spectroscopy. PFO displayed good solubility in ethanol, which was estimated to be at least $2 \mathrm{~g} \mathrm{~L}^{-1}$ at room temperature. In contrast, $\mathrm{PFO}$ was found to be non-soluble in water-based buffers. The good miscibility of ethanol and water permitted us to record the absorption and emission spectra of dissolved and aggregated PFO through solvent exchange. Aggregation resulted in absorption redshifting and considerable broadening of the spectrum. These results indicate that the polyfluorene structure and conformation are influenced by acidified water based conditions. Similar photophysical features have been attributed to formation of aggregates and reported previously for polyfluorenes ${ }^{9}$. We note that a gradual redshift in peak absorption by up to $50 \mathrm{~nm}$ is accompanied by a decrease in absorption strength and the appearance of a long tail above $500 \mathrm{~nm}$ which can be explained with light scattering by the aggregates (Fig $1 \mathrm{~b}$ ). We observe also a shoulder at $350-400 \mathrm{~nm}$ which arises due to the fraction of PFO that remains dissolved. The redshift of the absorption maximum occurs due to conformational changes of PFO such as straightening of the conjugated backbone and increase in conjugation length. Moreover, the non-solvent promotes intra-chain interactions such as $\pi$-stacking. To gain further insight into intramolecular interactions in PFO we performed photoluminescence experiments. Fig. 1c reveals a strong decrease in photoluminescence and a redshift upon addition of the non-solvent to the polyfluorene. We propose a two-step model of PFO aggregation where after addition of nonsolvent, in the first few minutes stiffening of chains and an increase in conjugation length occurs that could be explained by a distinct redshift in absorption and photoluminescence spectra. After this initial phase, gradual quenching of photoluminescence occurs due to $\pi$-stacking which is leading to aggregates with photophysical features that resemble those usually explained in terms of J-aggregation model used for small molecules. ${ }^{10-13}$

The next part of the study concerned the binding ability of both forms of PFO, dissolved and aggregated, to amyloid insulin fibrils. In particular, we employed UV-Vis absorption spectroscopy since binding of conjugated oligomers to amyloid fibrils is known to result in absorption shifting. Such an effect has been demonstrated previously for a number of polythiophene ${ }^{5}$ and polyfluorene derivatives ${ }^{6,14}$.

Fig. 2 presents absorption and linear dichroism spectra of dissolved and aggregated PFO complexed with insulin fibrils. Both (i) well-dissolved PFO in ethanol and (ii) aggregated PFO are stabilized by the presence of amyloid insulin fibrils and no time dependent absorption shifting was observed. In contrast,
PFO in $\mathrm{pH}=2$ buffer alone displays a rapid redshift of the peak absorption to more than $400 \mathrm{~nm}$ ( $c f$. Fig. 1b). Interestingly, when PFO aggregation was initiated through solvent exchange and the oligomer was allowed to aggregate for $20 \mathrm{~min}$ the addition of insulin fibrils promptly inhibited further spectral changes. These results indicate that insulin fibrils are able to stabilize the intermediate state of aggregation, as evidenced by the absence of any further redshift as well as the absence of any spectral changes (Fig. 2b). This observation is consistent with results obtained for dissolved PFO, for which attraction between the oligomer and amyloid fibrils is a driving force to form complexes.
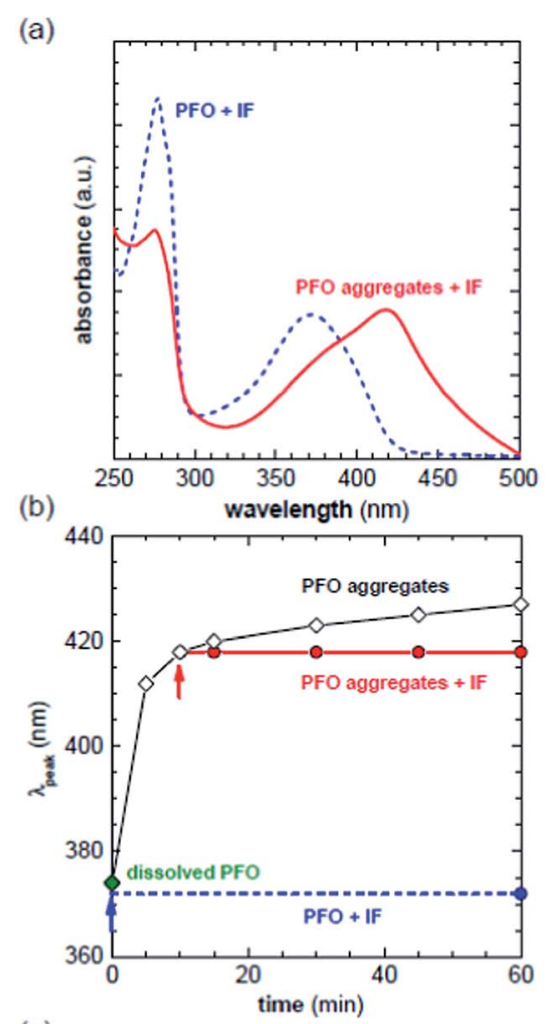

(c)

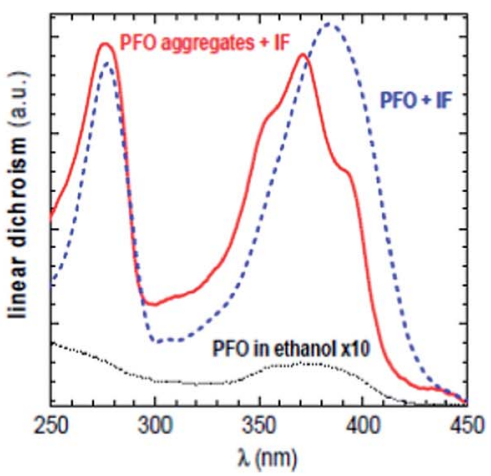

Fig. 2 Absorption spectra of dissolved (blue dashed) and aggregated PFO (red solid) with insulin fibrils (a); aggregation of pristine PFO: absorption maximum vs. time and the same measurements for dissolved and aggregated form upon binding to insulin fibrils (b); linear dichroism of dissolved (blue dashed), aggregated PFO (red solid) in dispersion of insulin fibrils. Reference of PFO in ethanol multiplied by a factor of 10 for the sake of comparison (c). 
To investigate the structure of PFO and its interactions with insulin fibrils in more detail linear dichroism (LD) was used (Fig 2c). PFO in ethanol only displays weak LD signals but features a strong positive band when complexed with insulin fibrils at $\mathrm{pH}=2$. The increase in LD amplitude is attributed to the rigidity and length of amyloid fibrils that orient better under flow. Hence, flow-aligned amyloid fibrils induce optical anisotropy in bound PFO. A positive LD signal suggests that PFO aligns with the oligomer backbone parallel to the long axis of the amyloid fibrils, which is consistent with previous reports. ${ }^{15}$ The same orientation was also observed for PFO that was first allowed to aggregate for $20 \mathrm{~min}$ followed by the addition of insulin fibrils, which arrest aggregation. Surprisingly, the LD spectrum features three distinct bands at $354 \mathrm{~nm}, 371 \mathrm{~nm}$ and $393 \mathrm{~nm}$, indicating subtle structural changes in the oligomer backbone when compared with well dissolved PFO bound to fibrils. Similar effects were reported for small molecules that form J-aggregates. ${ }^{16}$ We conclude that the observed spectral features are related to the aggregation rate where stiffening of chains and $\pi$-stacking interactions influence the photophysics of the PFO oligomer. The LD, which provides information on the transition dipole moments in aligned molecules, is sensitive to structural changes that can occur during the aggregation process. Thus the spatial confinement of the intermediate states of PFO aggregates on the fibril surface enables control over the oligomer structure, which facilitates monitoring of the photophysical properties by absorption spectroscopy.

We conclude that attractive forces between amyloid fibrils and PFO outweigh any tendency for inter- or intrachain coupling of PFO that occur upon solvent exchange. The most plausible binding mode is that both forms of PFO, dissolved in ethanol and aggregated, align in the channels at the fibrils surface since interior binding sites are sterically hindered ${ }^{\mathbf{1 7}}$ and the orientation of the oligomer is driven by the rate of interactions with amyloid fibrils. Since there are no protonated functional groups, it is feasible that supramolecular interactions occur due to $\pi-\pi$ stacking between aromatic residues and polyfluorene rings, which is maximized by the planar alignment of PFO at the surface. Ethylene glycol side chains may represent a further stabilizing factor, since they possess the ability to form hydrogen bonds with amino acids ${ }^{18}$ in between protofilaments of the fibrils and act as anchors. Since the aggregates are promptly stabilized by insulin fibrils it can be deduced that wellordered $\beta$-sheet structures act as nanotemplates for aggregating oligomers in solution. The presented results may be interpreted within the $\mathrm{HJ}$-aggregation ${ }^{\mathbf{1 0}}$ model, which seems to be most appropriate for describing amyloid-PFO system.

Inter- and intramolecular interactions that occur during aggregation of PFO as well as complexation with amyloid fibrils can lead to cooperative effects that tend to strongly influence the nonlinear absorption properties. ${ }^{19}$ In order to investigate whether such effects can be observed in case of PFO aggregates stabilized on amyloid fibrils, we used the open-aperture Z-scan technique, which can be employed to determine the nonlinear absorption coefficient $(\beta)$ (for details see Experimental section and ESI in ref. 20). The Z-scan technique is well-suited for determining TPA parameters in weakly emissive systems such as oligomer aggregates ${ }^{\mathbf{2 1}}$ or amyloid protein fibrils ${ }^{\mathbf{2 0}}$, where methods based on measurements of TPA induced emission may not be suitable. Thus, we used this setup to explore whether PFO and its aggregates display an enhanced TPA signal when complexed with amyloid fibrils. Z-scans were performed at $800 \mathrm{~nm}$, which is a suitable compromise wavelength for studying TPA of dissolved and aggregated PFO that one-photon absorption maximum is around $400 \mathrm{~nm}$. Moreover, in this region amyloid fibrils themselves absorb only through a three-photon process, ${ }^{20}$ which is a several orders of magnitude weaker effect and thus did not noticeably influence the nonlinear absorption properties of PFO at $800 \mathrm{~nm}$. Fig. 3a shows open aperture (OA) Z-scan traces for PFO in ethanol and theoretical fits that were produced by a custom program that used equations derived by Sheik-Bahae et $a .^{22}$ A detectable TPA signal was recorded for PFO and the nonlinear absorption strength was quantified as the value of the two-photon cross section $\sigma_{2}$, given in GM units where $1 \mathrm{GM}=$ $10^{-50} \mathrm{~cm}^{4} \mathrm{~s}_{\text {photon }}{ }^{-1}$. For the PFO stock solution of $2 \mathrm{~g} \mathrm{~L}^{-1}$ the calculated TPA is $\sigma_{2}=280 \mathrm{GM}$ per monomer unit. However, in order to measure a TPA that is attributed only to conformational changes of PFO and furthermore to complexation with fibrils, the detection threshold for PFO in ethanol was determined by lowering the oligomer concentration to $0.5 \mathrm{~g} \mathrm{~L}^{-1}$ where no OA signal was recorded. The same concentration was used for further experiments where in a first step PFO aggregates were formed by keeping oligomer in acidic water buffer for $20 \mathrm{~min}$. Since aggregates are unstable without the presence of amyloid fibrils but precipitate, it was not feasible to record a corresponding two-photon absorption spectrum. Instead, the solution becomes turbid and strong laser light rapidly overheats the sample at the focal spot, which results in a sharp peak decrease in an OA measurement (Fig. S5 $\dagger$ ). Similar experiments were performed with insulin fibrils, which stabilizes both the dissolved and aggregated form of PFO. Fig. $3 \mathrm{~b}$ shows open aperture (OA) Z-scan traces for diluted PFO in dissolved and aggregated form bound to fibrils. Some uncertainty can arise from aggregate scattering since the theoretical fit does not ideally match the experimental trace. Nevertheless, we observe a pronounced difference between data for dissolved and aggregated PFO attached to the fibrils surface. For the dissolved PFO bound to insulin fibrils the calculated TPA was $\sigma_{2}=350 \mathrm{GM}$ whereas a significantly stronger signal for aggregated PFO-insulin fibrils complexes yielded a four times higher value of $\sigma_{2}=1380 \mathrm{GM}$. This observation indicates that straightening of the conjugated backbone and stiffening of chains due to binding with amyloids leads to enhanced and sizable TPA, which can be attributed to through-bond cooperative effects. In case of aggregated PFO bound to insulin fibrils, through-space cooperativity can lead to further substantial enhancement of TPA signal. Thus both effects can contribute to the observed increase in TPA.

Reported values indicate that the use of conjugated polymers is advantageous as compared to standard chromophores such as ThT or Congo red in terms of two-photon absorption cross section, which is an important factor with regard to imaging techniques based on nonlinear optics. Whereas it is known that $\pi$-conjugation leads to a sizable increase by through-bond interactions, here we show for the first time that upon 
(a)

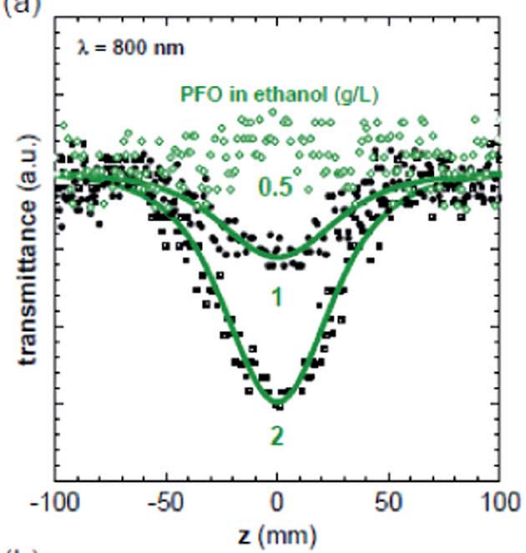

(b)

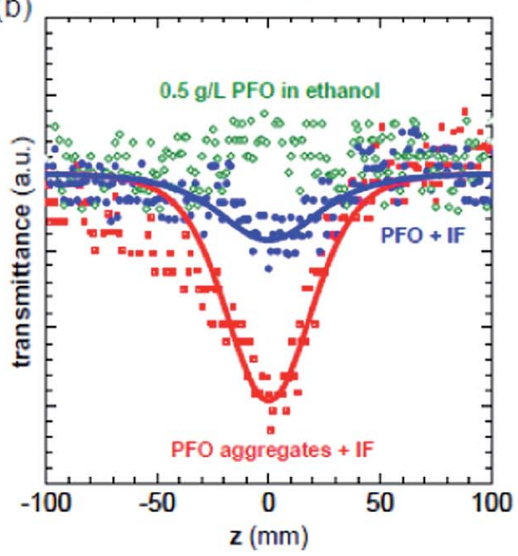

Fig. 3 Open aperture Z-scans representing two-photon absorption of PFO of different concentration in ethanol (a); PFO with insulin fibrils in the dissolved (blue dots) and aggregated (red squares) form. The reference sample was diluted PFO dissolved in ethanol (open green diamonds) (b)

aggregation additional enhancement occurs due to a close proximity of aggregated $\pi$-conjugated polymer on the fibril surface. Compared to small molecules used as amyloid staining agents where the two-photon absorption cross section is $\sim 50 \mathrm{GM}$, aggregated polyfluorene shows more than an order of magnitude higher values reaching nearly $1400 \mathrm{GM}$.

\section{Conclusions}

In summary, photophysical and optical properties of a polyfluorene derivative (PFO) and its binding to amyloid insulin fibrils were examined. Complexation based on weak supramolecular interactions between amyloid fibrils and PFO in dissolved and aggregated form led to sizable enhancement of twophoton absorption. Amyloid fibrils were used as nanotemplates that facilitate studies of nonlinear absorption cooperative effects in conjugated oligomers by stabilizing the intermediate states of aggregating molecules in solution.

\section{Acknowledgements}

P. H., D. A. G and C. M. acknowledge financial support from Chalmers Area of Advance - Nanotechnology, the Swedish
Research Council and Formas. P. H. and M. S. acknowledge support from the Foundation for Polish Science (Mistrz grant). Sandra Rocha and Anders Mårtensson (Chalmers) are thanked for the help with the linear dichroism and GPC measurements, respectively.

\section{References}

1 C. Zhu, L. Liu, Q. Yang, F. Lv and S. Wang, Water-Soluble Conjugated Polymers for Imaging, Diagnosis, and Therapy, Chem. Rev., 2012, 128(8), 4687-4735.

2 T. Klingstedt and K. P. R. Nilsson, Luminescent conjugated poly- and oligo-thiophenes: optical ligands for spectral assignment of a plethora of protein aggregates, Biochem. Soc. Trans., 2012, 40(4), 704-710.

3 L. De Boni, R. D. Fonseca, K. R. A. Cardoso, I. Grova, L. Akcelrud, D. S. Correa, et al. Characterization of twoand three-photon absorption of polyfluorene derivatives., $J$. Polym. Sci., Part B: Polym. Phys., 2014, 52(11), 747-754.

4 A. Åslund, C. J. Sigurdson, T. Klingstedt, S. Grathwohl, T. Bolmont and D. L. Dickstein, et al. Novel Pentameric Thiophene Derivatives for in Vitro and in Vivo Optical Imaging of a Plethora of Protein Aggregates in Cerebral Amyloidoses, ACS Chem. Biol., 2009, 4(8), 673-684.

5 K. P. R. Nilsson, A. Herland, P. Hammarstrom and O. Inganas, Conjugated Polyelectrolytes: ConformationSensitive Optical Probes for Detection of Amyloid Fibril Formation, Biochemistry, 2005, 44(10), 3718-3724.

6 A. Herland, D. Thomsson, O. Mirzov, I. G. Scheblykin and O. Inganäsa, Decoration of amyloid fibrils with luminescent conjugated polymers, J. Mater. Chem., 2008, 18(1), 126-132.

7 Y. Compta, L. Parkkinen, P. Kempster, M. Selikhova, T. Lashley and J. L. Holton, et al. The significance of $\alpha$ synuclein, amyloid- $\beta$ and tau pathologies in Parkinson's disease progression and related dementia, Neurodegener. Dis., 2014, 13, 154-156.

8 M. W. Head and J. W. Ironside, Review: Creutzfeldt-Jakob disease: prion protein type, disease phenotype and agent strain, Neuropathol. Appl. Neurobiol., 2012, 38(4), 296-310.

9 J. Teetsov and M. A. Fox, Photophysical characterization of dilute solutions and ordered thin films of alkyl-substituted polyfluorenes, J. Mater. Chem., 1999, 9(9), 2117-2122.

$10 \mathrm{H}$. Yamagata and F. C. Spano, Strong Photophysical Similarities between Conjugated Polymers and Jaggregates, J. Phys. Chem. Lett., 2014, 5(3), 622-632.

11 F. C. Spano and C. Silva, H- and J-Aggregate Behavior in Polymeric Semiconductors, Annu. Rev. Phys. Chem., 2014, 65(4), 477-500.

12 S. Giri, C. H. Moore, J. T. Mcleskey and P. Jena, Origin of Red Shift in the Photoabsorption Peak in MEH-PPV Polymer, $J$. Phys. Chem. C, 2014, 118(25), 13444-13450.

13 M. J. M. Wirix, P. H. H. Bomans, H. Friedrich, N. A. J. M. Sommerdijk and G. de With, ThreeDimensional Structure of P3HT Assemblies in Organic Solvents Revealed by Cryo-TEM, Nano Lett., 2014, 14(4), 2033-2038. 
14 D. Ghosh, P. Dutta, C. Chakraborty, P. K. Singh, A. Anoop and N. Nath Jha, et al. Complexation of Amyloid Fibrils with Charged Conjugated Polymers, Langmuir, 2014, 30(13), 3775-3786.

15 J. Wigenius, M. R. Andersson, E. K. Esbjörner and F. Westerlund, Interactions between a luminescent conjugated polyelectrolyte and amyloid fibrils investigated with flow linear dichroism spectroscopy, Biochem. Biophys. Res. Commun., 2011, 408(1), 115-119.

16 B. Hv, K. Ludwig and C. Böttcher, Pinacyanol chloride forms mesoscopic $\mathrm{H}$-and J-aggregates in aqueous solution-a spectroscopic and cryo-transmission electron microscopy study, Phys. Chem. Chem. Phys., 2014, 16(22), 10659-10668.

17 P. Hanczyc, Binuclear ruthenium(II) complexes for amyloid fibrils recognition, Chem. Phys., 2014, 445(12), 1-4.

$18 \mathrm{~S}$. Amani and A. Naeem, Transition of transferrin from native to fibrillar state: An implication for amyloid-linked diseases, Biochem. Eng. J., 2014, 91(15), 120-128.
19 E. Collini, Cooperative effects to enhance two-photon absorption efficiency: intra- versus inter-molecular approach, Phys. Chem. Chem. Phys., 2013, 14(11), 3725-3736.

20 P. Hanczyc, M. Samoc and B. Norden, Multiphoton absorption in amyloid protein fibres, Nat. Photonics, 2013, 7(12), 969-972.

21 J. Szeremeta, R. Kolkowski, M. Nyk and M. Samoc, Wavelength Dependence of the Complex Third-Order Nonlinear Optical Susceptibility of Poly(3-hexylthiophene) Studied by Femtosecond Z-Scan in Solution and Thin Film, J. Phys. Chem. C, 2013, 117(49), 26197-26203.

22 M. Sheik-Bahae, A. A. Said, T. Wei, D. J. Hagan and E. W. Stryland, Sensitive Measurement of Optical Nonlinearites Using a Single Beam, IEEE J. Quantum Electron., 1990, 26(4), 760-769. 